\title{
Clinical Significance of the Electrophysiologic Study (EPS)- Guided Therapy for the Secondary Prevention of Ventricular Tachycardia
}

\author{
Masaru Yuge, MD; Shinichi Niwano, MD; Masahiko Moriguchi, MD; Takeshi Sasaki, MD; \\ Shoji Hirasawa, MD; Ryuta Imaki, MD; Daisuke Sato, MD; Tohru Izumi, MD
}

\begin{abstract}
Background Although electrophysiologic study (EPS) is one of the most reliable methods for selecting preventive therapy for patients with sustained ventricular tachycardia (VT), VT may recur during EPS-guided effective therapy; therefore, the importance of implantable cardioverter-defibrillator (ICD) has been emphasized. In this study, the prognoses of VT patients were evaluated to clarify the importance of EPS-guided therapy for the secondary prevention of VT.

Methods and Results The study population consisted of 99 consecutive patients with a history of sustained VT, which was inducible in EPS. The VT induction protocol used 1-3 extrastimuli and rapid ventricular pacing at 2 right ventricular sites and included additional isoproterenol infusion. ICD implantation was applied to all patients with an episode of hemodynamically unstable VT, regardless of the result of preventive therapy. For preventive therapy, an antiarrhythmic drug and/or catheter ablation were selected, and they were defined as being effective in the EPS-guided therapy when the induction of VT was completely prevented. When no therapy was effective for prevention, an antiarrhythmic drug was prescribed under ICD implantation. During the follow-up period of $19 \pm 20$ months, VT recurred in 17 of 32 patients (53\%) in the ineffective group and in 10 of 67 patients $(15 \%)$ in the effective group ( $\mathrm{p}=0.0001)$. The therapies used in the effective group were class I antiarrhythmic drug in 9, class III in 15, and catheter ablation in 35 patients. Between the patients with and without VT recurrence, there were no significant differences in the left ventricular ejection fraction and the maximum number of repetitive ventricular responses that remained in VT induction in EPS.

Conclusions Although VT may recur in up to 15\% of patients with EPS-guided effective therapy, the recurrence rate was significantly reduced in comparison to that in the ineffective group. EPS-guided therapy may be useful to reduce the clinical recurrence of VT, as well as the action of ICD. (Circ J 2006; 70: 268-272)
\end{abstract}

Key Words: Antiarrhythmic therapy; Electrophysiologic study; Ventricular tachycardia

A lthough electrophysiologic study (EPS) is one of the most reliable methods for selecting preventive therapy for sustained ventricular tachycardia (VT), it has been reported that VT may recur in up to $8-25 \%$ of patients, even during EPS-guided effective therapy!-11 The recurrence rate can be reduced by using more complicated criteria for the selection of preventive therapy. As a result, the importance of an implantable cardioverter-defibrillator (ICD) has been emphasized, and the Antiarrhythmics Versus Implantable Defibrillators study has proved the superiority of ICD in comparison with amiodarone therapy in the secondary prevention of VT4 Other mega-trials, that is, MADIT-I, MUSTT, have shown the importance of EPS for the evaluation of patient risk; these trials did not examine the selection of preventive therapy in primary prevention of VT5,6 According to these results, the modern electrophysiologists tend to choose ICD first, and after that, do not usually pay strong attention to the preventive therapy. However, some patients suffer clinical VT recurrence, that

(Received July 25, 2005; revised manuscript received December 12, 2005; accepted December 19, 2005)

Department of Angio-Cardiology, Kitasato University School of Medicine, Sagamihara, Japan

Mailing address: Masaru Yuge, MD, Department of Angio-Cardiology, Kitasato University School of Medicine, 1-15-1 Kitasato, Sagamihara 228-8555, Japan. E-mail: yuge@pg7.so-net.ne.jp is, from the action of ICD, so that prevention is an important issue in therapeutic modality. Although, previous studies have documented the superiority of amiodarone to sodium-channel blockers, the importance of preventive therapies including catheter ablation as the secondary preventive therapy are not well understood. In the present study, the significance of EPS-guided selection of preventive therapy including catheter ablation in patients with sustained VT was evaluated and compared with non-applicable EPS guided pharmacological and/or ICD therapy.

\section{Methods}

Subjects

There were 130 consecutive patients selected with clinically documented sustained VT or ventricular fibrillation (VF) satisfying the following criteria: (1) more than one episode of VT/VF that was electrocardiographically documented; (2) the patient had undergone EPS for the evaluation of VT/VF; and (3) clinical episodes of VT/VF were reproducible by programmed electrical stimulation in EPS. Because idiopathic VT/VF cases including long QT or Brugada syndrome have different clinical characteristics from VT/VF cases with organic heart disease, they were excluded from the population. Finally, 99 VT/VF patients with organic heart disease were evaluated in the present 
Table 1 Clinical Characteristics of VT/VF Patients

\begin{tabular}{lcc}
\hline \hline & $V T$ & $V F$ \\
\hline$N$ (case) & 90 & 9 \\
Age (years) & $57.9 \pm 16.5$ & $56.2 \pm 12.4$ \\
Basic heart disease (case) & & \\
IHD & 41 & 8 \\
CM & 32 & 0 \\
VHD & 10 & 1 \\
Other & 7 & 0 \\
LVEF $(\%)$ & $46.7 \pm 14.7$ & $45.6 \pm 15.0$ \\
\hline
\end{tabular}

$V T$, ventricular tachycardia; $V F$, ventricular fibrillation; $I H D$, ischemic heart disease; CM, cardiomyopathy; VHD, valvular heart disease; LVEF, left ventricular ejection fraction.

study. The mean age was $58 \pm 16$ years old; 9 were female and 90 were male. The basic structural heart disease was ischemic heart disease in 49 patients, dilated or hypertrophic cardiomyopathy in 32 patients, valvular heart disease in 11 patients, the other heart diseases in 7 patients (congenital heart disease in 5, and sarcoidosis in 2). Out of 99 patients, the clinically documented arrhythmia was VT in 90 and VF in the remaining 9 patients. These clinical characteristics are summarized in Table 1.

\section{EPS}

Induction Protocol EPS was performed after obtaining written informed consent from all patients. All cardioactive agents were discontinued for a period 5 times longer than the half-life of each agent before the study (no amiodarone case was included). The induction protocol for VT/VF used 1-3 extrastimuli with 2 basic drive cycle lengths (400 and $600 \mathrm{~ms}$ ) and rapid ventricular pacing at fixed cycle lengths delivered at 2 right ventricular sites, $, 7,8,11$ When VT/VF could not be induced by electrical stimuli alone, isoproterenol was infused intravenously and the entire stimulation protocol was repeated. To eliminate the induction of non-specific arrhythmias, coupling intervals of extrastimuli $<180 \mathrm{~ms}$ were not delivered, even when they could capture the ventricle. The end-point of the induction protocol was the induction of sustained VT/VF. In EPSguided evaluation, preventive therapy was considered effective when VT/VF was induced prior to, but became uninducible with the whole induction protocol after the intervention. In this decision of effectiveness, the repetitive ventricular responses (RVRs) were allowed to remain up to 5 beats, but if there were 6 or more RVRs, this was considered ineffective?,12

Basic Strategy for the Arrhythmias First, the patients with hemodynamically unstable VT/VF episodes were considered principal candidates for ICD implantation regardless of the result of the EPS-guided evaluation. In EPS, reproducibility, that is, the induction of clinical VT/VF, was attempted by using the standard VT/VF induction protocol as described above. In patients with inducible monomorphic VT, catheter ablation was applied for all VT foci including non-clinical QRS morphology. To determine the ablation sites, endocardial mapping, pace-mapping and/or substrate mapping were used as the standard technique? Catheter ablation was considered effective when all VT/VFs became uninducible after the ablation, and they were followed up without the prescription of any antiarrhythmic agents. When the catheter ablation was not effective for prevention of VT induction, one of the antiarrhythmic agents available for intravenous infusion was chosen and its effect
Table 2 Clinical Characteristics of Patients Divided by EPS-Guided Therapy

\begin{tabular}{|c|c|c|c|}
\hline & $\begin{array}{l}\text { Effective } \\
\text { group }\end{array}$ & $\begin{array}{l}\text { Ineffective } \\
\text { group }\end{array}$ & $p$ value \\
\hline$N($ case $)$ & 67 & 32 & \\
\hline Male sex (\%) & $86.1 \%$ & $96.9 \%$ & 0.154 \\
\hline Age (years) & $57.9 \pm 16.3$ & $57.5 \pm 16.0$ & 0.913 \\
\hline Basal heart disease (case) & $\begin{array}{l}\text { IHD (28), } \\
\text { CM (23), } \\
\operatorname{VHD}(10), \\
\text { Other (6) }\end{array}$ & $\left.\begin{array}{c}\operatorname{IHD}(21), \\
C M(9), \\
\operatorname{VHD}(1), \\
\text { Other (1) }\end{array}\right]$ & -0.221 \\
\hline \multicolumn{4}{|l|}{ Preventive therapy (case) } \\
\hline Class I & 8 & 4 & \\
\hline Class III & 15 & 11 & \\
\hline Catheter ablation & 35 & 9 & $-0.0239 *$ \\
\hline Other & 9 & 8 & \\
\hline $\operatorname{LVEF}(\%)$ & $49.3 \pm 2.0$ & $41.0 \pm 2.7$ & $0.0229 *$ \\
\hline ICD (case) & 7 & 18 & \\
\hline
\end{tabular}

EPS, electrophysiologic study; ICD, implantable cardioverter-defibrillator. Other abbreviations see in Table 1.

I, III, class name in Vaughan-Williams antiarrhythmic drug classification.

for prevention of VT induction was evaluated in the following EPS. The effect of this combination therapy was evaluated in the same manner. When the drug was effective, the same antiarrhythmic agents were continued orally. This preventive trial of pharmacological therapy was evaluated only once in each patient, that is, a single drug per patient, to reduce costs in terms of repetition of EPS or prolongation of hospital admission. When the drug was ineffective for prevention of VT induction, ICD implantation was chosen as the basic therapy. If VT recurred frequently, pharmacological therapy was additionally chosen conventionally, that is, amiodarone or the other agents in accordance with clinical effects, such as interruption of VT. If VT recurred rarely, they were observed just using ICD therapy. In patients with inducible VF or sustained polymorphic VT, ICD was chosen as the basic therapy and additional preventive therapy with antiarrhythmic agents was chosen based on the clinical situation of each patient, that is, mainly class Ib, class III antiarrhythmic agents for patients with left ventricular (LV) dysfunction or ischemic heart diseases in the chronic phase. When patients did not have severe LV dysfunction and a history of congestive heart failure, a class I antiarrhyhtmic agent was chosen. The effect of antiarrhythmic agents for prevention of VF induction was evaluated in the following EPS.

\section{Clinical Follow up}

The patients were divided into 2 groups based on the results of EPS-guided evaluation, that is, the effective and ineffective groups. The clinical characteristics of the 2 groups are summarized in Table 2 . In the effective and ineffective groups, the prognoses of the patients, that is, sudden cardiac death and/or arrhythmic events, were evaluated during clinical follow up. Several clinical parameters, such as underlying heart disease, $\mathrm{LV}$ dysfunction, types of therapies, and number of RVRs remaining in the EPS, were compared between patients with and without clinical events in each group.

\section{Statistical Analysis}

Values are presented as mean \pm standard deviation. Statistical analysis was performed by using the Wilcoxon test in the Kaplan-Meier survival curve or t-test. A p $<0.05$ was 


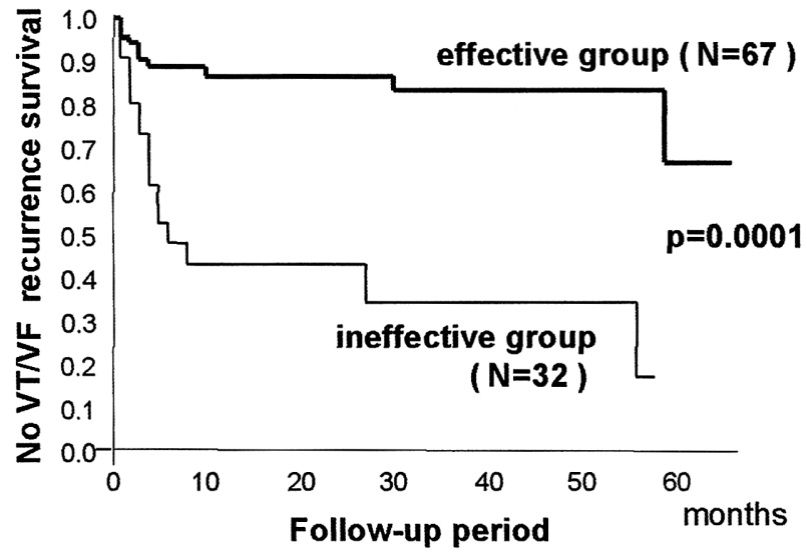

Fig 1. Recurrence rate of ventricular tachycardia/ventricular fibrillation (VT/VF) in electrophysiologic study (EPS)-guided effective and ineffective groups. VT/VF recurrence was observed in 17 of 32 patients $(53 \%)$ in the EPS-guided ineffective group, and in 10 of 67 $(15 \%)$ in the effective group. The recurrence rate was significantly lower in the effective group $(\mathrm{p}=0.0001)$.

Table 3 Clinical Characteristics of Effective Group

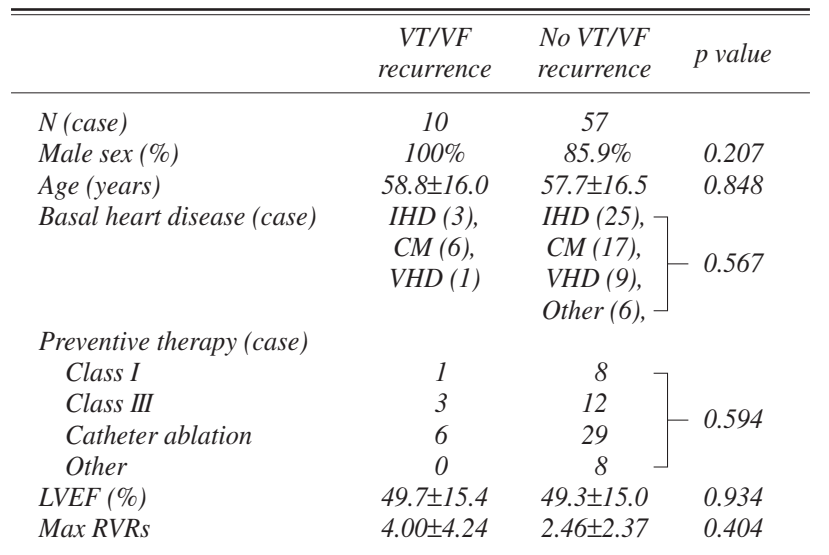

$R V R s$, repetitive ventricular responses. Other abbreviations see in Tables 1 and 2 .

considered significant.

\section{Results}

The study population consisted of $90 \mathrm{VT}$ and $9 \mathrm{VF}$ patients. The clinical characteristics of VT/VF are summarized in Table 1. There was no significant difference between the 2 groups. Table 2 shows the clinical characteristics of the effective group and ineffective group in EPSguided evaluations. There was no significant difference in the clinical parameters except for the LV ejection fraction (LVEF) and the population of patients with catheter ablation. LVEF was significantly higher in the effective group than in the ineffective group $(\mathrm{p}=0.0239)$. VT recurrence was observed in 17 of 32 patients $(53 \%)$ in the ineffective group, and in 10 of 67 patients (15\%) in the effective group (Fig 1; $\mathrm{p}=0.0001$ ). In most patients with VT/VF recurrence, sudden cardiac death was prevented by ICD, with the exception of 2 patients in the ineffective group (6.3\%; Fig 2; $\mathrm{p}=0.002$ ).

Tables 3 and 4 show the comparison between patients with and without VT/VF recurrence in each of the 2

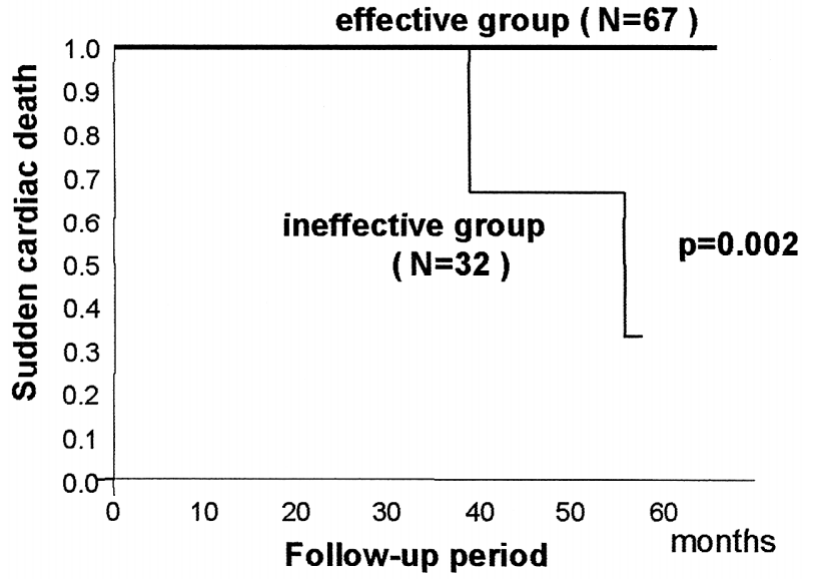

Fig 2. Rate of sudden cardiac death in electrophysiologic study (EPS)-guided effective and ineffective groups. Sudden cardiac death was observed in 2 patients $(6.3 \%)$ in the EPS-guided ineffective group, and none in the effective group. There was a significant difference between the 2 groups $(\mathrm{p}=0.002)$.

Table 4 Clinical Characteristics of Ineffective Group

\begin{tabular}{|c|c|c|c|}
\hline & $\begin{array}{c}V T / V F \\
\text { recurrence }\end{array}$ & $\begin{array}{l}\text { No VT/VF } \\
\text { recurrence }\end{array}$ & $p$ value \\
\hline$N$ (case) & 17 & 15 & \\
\hline Male sex (\%) & $94.1 \%$ & $100 \%$ & 0.339 \\
\hline Age (years) & $56.2 \pm 13.9$ & $58.9 \pm 18.4$ & 0.641 \\
\hline Basal heart disease (case) & $\begin{array}{l}\operatorname{IHD}(10), \\
C M(5), \\
\operatorname{VHD}(1), \\
\text { Other }(1)\end{array}$ & $\left.\begin{array}{c}I H D(11) \\
C M(4)\end{array}\right]$ & -0.564 \\
\hline \multicolumn{4}{|l|}{ Preventive therapy (case) } \\
\hline Class I & 3 & 1 & \\
\hline Class III & 6 & 5 & \\
\hline Catheter ablation & 6 & 3 & 0.337 \\
\hline Other & 2 & $6 \perp$ & \\
\hline $\operatorname{LVEF}(\%)$ & $36.7 \pm 9.1$ & $45.9 \pm 15.1$ & 0.067 \\
\hline Max RVRs & $3.67 \pm 1.52$ & $7.00 \pm 2.16$ & 0.074 \\
\hline
\end{tabular}

$R V R s$, repetitive ventricular responses. Other abbreviations see in Tables 1 and 2 .

groups; that is, the EPS-guided effective and ineffective groups. None of the clinical parameters were significantly different between the patients with and without VT/VF recurrence in both the effective and ineffective groups. Although the maximum number of RVRs in the EPS was expected to be a marker for clinical VT/VF recurrence, this value was not significantly different between the patients with and without VT/VF recurrence in both of EPS-guided effective and ineffective groups.

One patient with a hemodynamically unstable VT episode was indicated for ICD implantation even though the case had EPS-guided effective antiarrhythmic therapy. In the other 6 patients with EPS-guided effective preventive therapies, ICD was indicated in accordance with the patients' selection.

\section{Discussion}

Clinical Importance of EPS-Guided Preventive Therapy

Although EPS is one of the most reliable methods for selecting preventive therapy for patients with sustained VT, VT recurrence may occur even during EPS-guided effec- 
tive therapy!-10 In cases with VF, the sensitivity and specificity of VF induction itself in EPS might be unreliable, therefore EPS-guided preventive therapy in VF is considered difficult9,13-15 In accordance with these results, cardiologists now tend to choose ICD therapy regardless of the effect of preventive therapy for VT/VF; however, prevention, or at least the reduction of clinical recurrence, is still an important issue for the management of VT/VF patients.,2,9,12,16-20 In the present study, we evaluated the long-term prognoses of patients with spontaneous episodes of VT/VF by attempting to choose preventive therapy under the guidance of EPS. In total, we observed VT/VF recurrence in 27 of 99 patients, but the event rate was significantly lower in the EPS-guided effective group (10 of $67,15 \%$ ) than in the EPS-guided ineffective group (17 of $32,53 \% ; \mathrm{p}=0.0001$ ), and the incidence of sudden cardiac death was significantly lower in the EPS-guided effective group ( $p=0.002$ ). This result seems to be similar to the earlier reports evaluating the usefulness of EPS-guided pharmacological therapy for VT/VF! $!^{-5,21,22}$ However, the basic strategy for the selection of preventive therapy was different. Because ICD therapy is now frequently used for most VT/VF patients, especially for those with an episode of syncope, repetition of EPS for the selection of preventive antiarrhythmic agents seems to be unnecessary and so that it should be avoided in the modern therapeutic strategy. In the present study, we tested only one antiarrhythmic agent for each patient, and therefore other effective antiarrhythmic agents for VT/VF prevention might have been overlooked. Yet the recurrence rate of VT/VF in the clinical courses was significantly lower in the effective group than in the ineffective group even in this simplified method. The incidence of catheter ablation as the final therapy was higher in the effective group than in the ineffective group. This probably relates to the severity of the basic heart disease, namely, less severe LV dysfunction might result in a smaller number of VT foci, which would be easier for successful ablation.

The simplified protocol in the present study might identify the patients with more benign VT/VF because the selection of the therapy was not randomized?,10,23 However, in clinical practice, this method of drug selection should be routine work in the EPS; the results in the present study indicate the benefit of EPS-guided preventive therapy as compared to blinded amiodarone therapy. Raczak et al reported EPS-guided therapy in patients who survived a sustained arrhythmic event following myocardial infarction is poorly predictive of future sudden death; however, their study did not include catheter ablation as the preventive therapy ${ }^{24}$ Considering the reported superiority of amiodarone to other antiarrhythmic agents, $5-27$ this result might be because of the effect of catheter ablation, especially for patients with monomorphic VTs. However, the VT recurrence rate was not significantly different between catheter ablation only (6 of 35) and the other preventive therapies (4 of 32) in the effective group ( $\mathrm{p}=0.594)$.

\section{Prediction of Clinical Recurrence of VT/VF}

It has been reported that a history of syncope, spontaneous non-sustained VT, and/or LV dysfunction are independent risk factors for the occurrence of clinical VT/VF'28-31 Other investigators have reported VT with non-clinical QRS morphology or multiple RVRs ( $>6$ beats) in EPS are other risk factors for $\mathrm{VT} / \mathrm{VF}$ recurrence,, 12 and non-invasive baroreflex sensitivity is of value in predicting VT/VF epi- sode recurrence ${ }^{24}$ However, in the present study, there was no significant difference in clinical parameters between patients with and without VT/VF recurrence in the EPSguided effective and ineffective groups. This result indicates the difficulty of the prediction of VT/VF recurrence even in patients under EPS-guided effective therapy. Therefore, ICD should be applied to patients with a history of syncope or hemodynamically unstable VT/VF regardless of the result in EPS-guided evaluation.

\section{Limitations}

The present study has several limitations. First, because the study was designed retrospectively, the true impact of EPS-guided preventive therapy in VT/VF patients was unclear. Second, we cannot make conclusions about patients with uninducible VT/VF in EPS. Third, because of the usage of various antiarrhythmic agents, the role of amiodarone in conventional preventive therapy was unclear. Fourth, a 19-month follow-up period might be too short to discuss the clinical importance of each therapeutic modality. Finally, because the number of patients was limited, the importance of various clinical parameters for VT/VF recurrence was unclear. These points should be re-evaluated and resolved in a future study.

\section{Conclusion}

In secondary prevention for patients with spontaneous and inducible VT/VF in EPS, EPS-guided preventive therapy including catheter ablation reduced the recurrence of VT/VF significantly. Although ICD therapy should be principally recommended for VT/VF patients, especially those with hemodynamically unstable episodes, EPS-guided preventive therapy was considered beneficial to reduce the VT/VF episodes as well as the action of ICD.

\section{Acknowledgments}

This study was supported, in part, by a grant-in-aid for scientific research for Dr Shinichi Niwano from the Postgraduate Research Project at Kitasato University, Kanagawa, Japan.

\section{References}

1. Wilber D, Garan H, Finkelstein D, Kelly E, Newell J, McGovern B, et al. Out-of-hospital cardiac arrest: Use of electrophysiologic testing in the prediction of long-term outcome. $N$ Engl J Med 1988; 318: $19-24$.

2. Niwano S, Yamaura M, Yoshizawa N, Moriguchi M, Kitano Y, Aizawa Y, et al. Electrophysiologic parameters to predict clinical recurrence of ventricular tachycardia in patients under electrophysiologic study guided effective pharmacological therapy. Jpn Circ J 1999; 63: 674-680.

3. Josephson ME, Horowitz LN. Electrophysiologic approach to therapy of recurrent sustained ventricular tachycardia. Am J Cardiol 1979; 43: $631-642$.

4. The Antiarrhythmics Versus Implantable Defibrillators (AVID) Investigators. A comparison of antiarrhythmic-drug therapy with implantable defibrillators in patients resuscitated from near-fatal ventricular arrhythmia. N Engl J Med 1997; 337: 1576-1583.

5. Buxton AE, Lee KL, DiCarlo L, Gold MR, Greer GS, Prystowsky $\mathrm{EN}$, et al. Electrophysiologic testing to identify patients with coronary artery disease who are at risk for sudden death. Multicenter Unsustained Tachycardia Trial Investigators. N Engl J Med 2000; 342: $1937-1945$.

6. Moss AJ, Hall WJ, Cannom DS, Daubert JP, Higgins SL, Klein H, et al for the Multicenter Automatic Defibrillator Implantation Trial Investigator. Improved survival with an implanted defibrillator in patients with coronary disease high risk for ventricular arrhythmia. $N$ Engl J Med 1996; 335: 1933-1940.

7. Niwano S, Aizawa Y, Satoh M, Chinushi M, Shibata A. Low energy electrical ablation for sustained ventricular tachycardia. Am Heart J 
1991; 119: $81-88$.

8. Aizawa Y, Chinushi M, Naitoh N, Kusano Y, Kitazawa H, Takahashi $\mathrm{K}$, et al. Catheter ablation with radiofrequency current of ventricular tachycardia originating from the right ventricle. Am Heart J 1993; 125: $1269-1275$.

9. Gillis AM, Sheldon RS, Wyse DG, Duff HJ, Cassidy MR, Mitchell LB. Clinical and electrophysiologic predictor of ventricular tachyarrhythmia recurrence in patients with implantable cardioverter defibrillators. J Cardiovasc Electrophysiol 2003; 14: 492-498.

10. Waller TJ, Kay HR, Spielman SR, Kutalek SP, Greenspan AM, Horowitz LN. Reduction in sudden death and total mortality by antiarrhythmic therapy evaluated by electrophysiologic drug testing: Criteria of efficacy in patients with sustained ventricular tachyarrhythmia. J Am Coll Cardiol 1987; 10: 83-89.

11. Toda H, Ito S, Naito S, Taniguchi K, Ueda M, Shinbo G, et al. Prevalence and electrocardiographic characteristics of idiopathic ventricular arrhythmia originating in the free wall of the right ventricular outflow tract. Circ J 2004; 68: 909-914.

12. Mitchell LB, Sheldon RS, Gillis AM, Connolly SJ, Duff HJ, Gardner MJ, et al. Definition of predicted effective antiarrhythmic drug therapy for ventricular tachyarrhythmias by the electrophysiologic study approach: Randomized comparison of patient response criteria. J Am Coll Cardiol 1997; 30: 1346-1353.

13. Raitt MH, Dolack GL, Kudenchuk PJ, Poole JE, Bardy GH. Ventricular arrhythmias detected after transvenous defibrillator implantation in patients with a clinical history of only ventricular fibrillation. Circulation 1995; 91: 1996-2001.

14. Poole JE, Mathisen TL, Kudenchuk PJ, McAnulty JH, Swerdlow $\mathrm{CD}$, Greene HL, et al. Long-term outcome in patients who survive out of hospital ventricular fibrillation and undergo electrophysiologic studies: Evaluation by electrophysiologic subgroups. J Am Coll Cardiol 1990; 16: 657-665.

15. Stevenson WG, Brugada P, Waldecher B, Zehender M, Wellens HJ. Clinical, angiographic and electrophysiologic findings in patients with aborted sudden death as compared with patients with sustained ventricular tachycardia after myocardial infarction. Circulation 1985; 71: 1146-1152.

16. Winkle RA, Mead RH, Ruder MA, Gaudiani VA, Smith NA, Buch WS, et al. Long-term outcome with the automatic implantable cardioverter-defibrillator. J Am Coll Cardiol 1989; 13: 1353-1361.

17. Moss AJ, Hall WJ, Cannom DS, Daubert JP, Higgins SL, Klein H, et al. Improved survival with an implanted defibrillator in patients with coronary disease at high risk for ventricular arrhythmia: Multicenter Automatic Defibrillator Implantation Trial Investigators. $N$ Engl $J$ Med 1996; 335: 1933-1940.

18. Zipes DP, Roberts D. Pacemaker-Cardioverter-Defibrillator Investigators: Results of the international study of the implantable pacemaker cardioverter-defibrillator: A comparison of epicardial and endocardial lead system. Circulation 1995; 92: 59-65.
19. The AVID Investigators. Antiarrhythmics versus implantable defibrillators (AVID): Rationale, design, and methods. Am J Cardiol 1995; 75: 470-475.

20. Bardy GH, Lee KL, Mark DB, Poole JE, Packer DL, Boineau R, et al; Sudden Cardiac Death in Heart Failure Trial (SCD-HeFT) Investigators. Amiodarone or an implantable cardioverter-defibrillator for congestive heart failure. N Engl J Med 2005; 352: 225-237.

21. Swerdlow CD, Peterson J. Prospective comparison of Holter monitoring and electrophysiologic study in patients with coronary artery disease and sustained ventricular tachyarrhythmias. Am J Cardiol 1985; 56: 577-580.

22. Aizawa Y, Murata M, Satoh M, Funazaki T, Matsuoka A, Shibata A, et al. Requirements of non-pharmacologic interventions in the treatment of recurrent sustained ventricular tachycardia. Jpn Circ J 1990; 54: $1340-1348$.

23. Niwano S, Furushima H, Taneda K, Abe A, Ohira K, Aizawa Y. The usefulness of Holter monitoring in selecting pharmacological therapy for patients with sustained monomorphic ventricular tachycardia: Studies in patients in whom no effective pharmacologic therapy could be determined by electrophysiologic study. Jpn Circ J 1998; 62: $347-352$.

24. Raczak G, Pinna GD, Maestri R, Danilowicz-Szymanowicz L, Szwoch M, Lubinski A, et al. Different predictive values of electrophysiological testing and autonomic assessment in patients surviving a sustained arrhythmic episode. Circ J 2004; 68: 634-638.

25. Aiba T, Kurita T, Taguchi A, Shimizu W, Suyama K, Aihara N, et al. Long-term efficiency of empirical chronic amiodarone therapy in patients with sustained ventricular tachyarrhythmia and structure heart disease. Jpn Circ J 2002; 66: 367-371.

26. Greene HL. The CASCDE study: Randomized antiarrhythmic drug therapy in survivors of cardiac arrest in Seattle: CASCADE investigators. Am J Cardiol 1993; 72: 70-74.

27. Doval HC, Nul DR, Grancelli HO, Perrone SV, Bortman GR, Curiel $\mathrm{R}$. Randomized trial of low-dose amiodarone in server congestive heart disease: Grupo de Estudio de la Sobrevida en la Insuficiencia Cardiaca en Argentina (GESICA). Lancet 1994; 344: 493-498.

28. Niwano S, Aizawa Y, Chinushi M, Tamura M, Ishiguro J, Ebe K, et al. Syncope and sudden cardiac death in patients with sustained ventricular tachycardia. Heart 1990; 22(Suppl): 43-48.

29. Brembilla-Perrot B, Suty-Selton C, Alla F, Beurrier D, Houriez P, Claudon O, et al. Risk factors for cardiac mortality in cases of syncope with previous history of myocardial infarction. Arch Mal Coeur Vaiss 2003; 96: $1181-1186$.

30. Buxton AE, Marchlinski FE, Waxman HL, Flores BT, Cassidy DM, Josephson ME. Prognostic factors in nonsustained ventricular tachycardia. Am J Cardiol 1984; 53: 1275-1279.

31. Swerdlow CD, Winkle RA, Mason JW. Determinants of survival in patients with ventricular tachyarrhythmias. N Engl J Med 1983; 308: $1436-1442$. 